"Spectrometric study of the oligodeoxyribonucleotide protonation in aqueous solution" Gargallo, R., Eritja, R., Kudrev, A.G. Russ. J. Gen. Chem., 80(3), 485-492 (2010). English translation. Zhurnal Obshchei Khimii, 80(3), 462-470 (2010). Original text in Russian.

doi: $10.1134 / \mathrm{S} 1070363210030205$

\title{
Spectrometric Study of the Oligodeoxyribonucleotide Protonation in Aqueous Solution
}

\author{
R. Gargallo, ${ }^{\text {a R. Eritja, }{ }^{b} \text { and A. G. Kudrev }}$ \\ University of Barcelona, Barcelona, Spain ' Institute of Structural Biology, \\ Barcelona, Spain ‘ St. Petersburg State University, Universitetskii pr. 26, St. \\ Petersburg, 198504 Russia- e-mail: kudrevandrei@mail.ru
}

\begin{abstract}
UV absorption spectra and circular dichroism spectra of aqueous solutions of cytosin- and thymine-containing single-stranded Oligodeoxyribonucleotide 5'-CCTTTCCTTTTCCTTTCC-3'(ckit4) were measured at various $\mathrm{pH}$ in the range 3.3-8.9. The chemometric analysis of the multiinstrumental data matrix was carried out. The diagrams of relative contents of complex forms of the DNA molecule absorbing in the studied wavelength range (220-320 nm) were constructed by the ALS-MCR soft simulation procedure without initial postulation of their chemical compositions. The model of equilibrium complex formation describing observed changes in the spectra depending on the solution acidity was developed on the basis of the matrix method. Intrinsic protonation constants of the oligonucleotide ckit4 were calculated. The formation of intramolecular complexes between cytosine $C . C^{+}$bases in the studied DNA molecule is of a cooperative nature, and their subsequent protonation is an anticooperative process.
\end{abstract}

DOI: $10.1134 / \mathrm{S} 1070363210030205$

Cooperativity of the ligand binding by a central molecule of a complex-forming agent plays a defining role in a number of biologically important processes [1]. A number of mathematical models have been developed to describe such interactions with infinite polymers [2-6]. The matrix method [7] has been offered for the calculation of equilibrium constants of the ligand cooperative binding by homogeneous oligomers. Alongside with so-called "rigid" simulation methods for the description of conformation and acid- base conversions of biopolymers, chemometric methods of the "soft" simulation [8-11] are widely used. The chemometric analysis is integrated in the MCR-ALS (Multivariate Curve ResolutionAlternating Least Squares) procedure [12]. To examine the possibility of simulating a cooperative complex formation, in the present work we have considered a specific example of folding a single-stranded oligomer with the formation of an intramolecular structure in the course of the cytosincontaining DNA protonation.

It is known that cytosine-rich DNA chains in weakly acid or even in neutral aqueous solutions can form tetraplexes, which bear the name $i$ motif [13-17].

The formation of the $i$-motif structure by the oligodeoxyribonucleotide 5'-CCTTTCCTITТСТПТCC-3' (ckit4), which was selected as the object of study in the present work, has been shown earlier by the NMR method [18]. 
It is supposed that in the case of ckit4 this structure consists of 4 locks organized from 2 parallel duplexes fastened in the antiparallel orientation. The structure is stabilized due to formation of intramolecular complexes between protonated and free cytosine bases C. $^{+}$(see scheme).

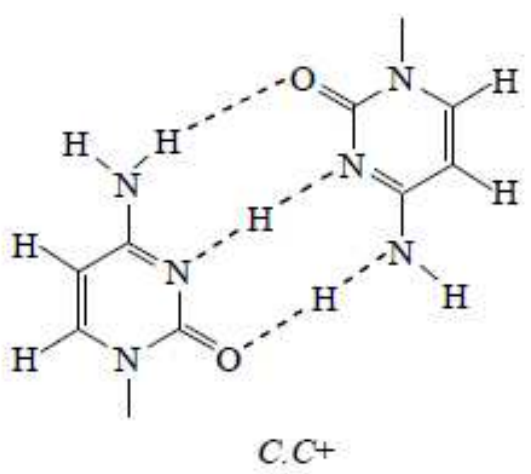

Synthetic oligomers of definite length and sequence have recently found a wide application as models of the polymeric DNA and RNA [19]. The oligonucleotides forming $i$-motif structures have a transcriptional activity in some genes and also find application in nanotechnologies [20-23]. Though $i$-motif structures are preferably formed in weakly acid solutions, there are proves of their existence in vivo. It is supposed that i-motif structures are formed in human chromosomes and in promoting locales of some genes [24].

The present work was fulfilled with the aim of developing a procedure for joint application of ALS- MCR and matrix simulation methods, taking, as a case in point, the study of the mechanism of protonation of a singlestranded DNA oligomer in an aqueous solution on the basis of spectrometric titration data.

Expansion of multivariable dependences by means of the MCR-ALS procedure [12]. The use of the chemometric analysis allows spectrometric data tj be described using so-called "soft model." The soft simulation of equilibrum interconversions consists in constructing diagrams of concentration profiles of abstract spectral forms participating in interconversions in a system. These profiles can be used to build the model of the system having a physical sense, so-called "rigid model." Parameters of a rigid model (equilibrium constants) in the case of a complex formation with non-polymeric ligands can be calculated from diagrams constructed with the use of evolving factor analysis [25-27]. It has been shown that in the case of a single-stranded polymer $[28,29]$ parameters of equilibrium reactions in a system with a concentration selectivity can be calculated immediately from the diagram constructed by means of ALS- MCR. In the absence of selectivity, the parameters of a complex formation involving a polydentate molecule are calculated from diagrams ALS-MCR with a constant error [30].

Calculation of intrinsic constants of the oligonucleotide protonation by a matrix method. In the present work the modified matrix model [7] was used to calculate equilibrium protonation constants of the oligomer under study. The basic allowance of the matrix model consists in the fact that the 
central molecule contains vacancies in fixed positions, which can add ligands. In the case under consideration the process is in essence the adsorption of protons by a DNA molecule caused by the presence of specific functional groups. In a neutral medium the molecule has the conformation of a stretched helix, and when pH decreases, the molecule is rolled up due to formation of intramolecular hydrogen bonds between protonated and neutral cytosine bases (see scheme). Thus an intramolecular complex is formed, in which half of the bound bases is capable to add protons, but constants of their protonation differ from the corresponding constants for the free bases in a neutral medium.

According to the matrix method, the model of the complex formation with an oligomer, capable of essential variation of its molecular structure depending on the complex formation degree, can be presented as follows. Let complexes $\left[\mathrm{PnH}_{n}\right]^{n+}(n=0,1,2, \ldots, N)$ are formed in equilibrium conditions by the addition of ligands (in our case of protons) to vacancies of a polynucleotide molecule (Pn). The whole set of complexes is present irrespective of concentrations of components, and the relative oligomer distribution between forms is defined by the stability of corresponding compounds. The set of constants describing stability of each configuration of $\left[\mathrm{PnH}_{n}\right]^{n+}$ complexes can be presented in the form of the product of equilibrium constants of ligand addition to separate vacancies $\left(\beta_{k i}\right)$ [Eq. (1)].

$$
\mathrm{B}_{k}=\prod_{i}^{N} \beta_{k}
$$

To calculate $\beta_{k i}$, we shall introduce a matrix of configurations $\mathbf{M}\left(2^{N}\right.$, $N)$. A matrix row $M_{k}$ reproduces one of possible configurations of the complex with a sequence of zeros in positions with free vacancies, and with a sequence of unities in positions with vacancies occupied by ligands. The expression for the equilibrium constant of a ligand addition to a particular vacancy looks like Eq. (2).

$$
\beta_{k i}=\left(M_{k i} \bar{K} \omega_{k i}\right)_{M_{k i} \neq 0} .
$$

Here $K^{-}=K_{\text {in }}$ is an intrinsic equilibrium constant of the reaction of the first ligand (proton) addition to a central molecule and $\omega_{k i}$ is a correction for mutual influence. In the present work we have considered the instance when three independent variables $K_{\text {in, }}, \omega_{c}$, and $\omega_{a}$ are necessary to describe the whole process. In our case it is accepted that four forms are formed: free polynucleotide I, molecule II with one proton added, partially protonated form III, which has formed an i-motif intramolecular complex, and form IV, which has lost the i-motif structure. When a secondary structure is formed each constant of a vacancy protonation is multiplied by $\omega_{c}$. When the intramolecular complex is protonated the constant is additionally multiplied by $\omega_{a}$. The $i$-motif structure is lost when the number of protonated intramolecular pairs is greater than a half of the total number of added protons. To summarize concentrations of $\left[\mathrm{PnH}_{n}\right]^{n+}$ in various configurations falling into to a particular form, we shall introduce a matrix $\mathbf{P}\left(2^{N}, 4\right)$. Elements of this matrix are equal to $N$ in the column corresponding to the selected form, and the other elements are equal to 
zero. Now we can calculate the matrix of fractions of equilibrium forms $\left[\mathrm{PnH}_{n}\right]^{n+}$ [Eq. (3)] for a particular solution $\mathrm{pH}$.

$$
\begin{gathered}
c_{\text {form }}=\frac{\left\{\left(\bar{K}^{s} \cdot \omega\right)^{T} \cdot[H]^{s}\right\}^{T}}{\bar{K}^{s} \cdot \omega \times[H]^{s}} \times \mathrm{P} \\
=\left(\frac{1}{S S} ; \frac{\bar{K} \cdot[H]}{S S} ; \ldots \frac{\omega_{i} \bar{K}^{i} \cdot[H]^{i}}{S S} ; \ldots\right) \times \mathrm{P} .
\end{gathered}
$$

Here $S S=K^{s} \omega \times[H]^{s}=\Sigma\left(\bar{K}^{s} \omega_{s} \cdot[H]^{s}\right) ; \bar{K}^{s}=\left[1, \bar{K}, \bar{K}^{2}, \ldots\right.$, $\left.K^{N}\right] ;[\mathrm{H}]^{S}=\left[1,[\mathrm{H}],[\mathrm{H}]^{2}, \ldots,[\mathrm{H}]^{N}\right]^{T} ;[\mathrm{H}]$

is the equilibrium concentration of protons; $s=[0,1,2, \ldots, N] ; \omega$ is the correction for mutual influence; $\times$ and $T$ are signs of elementwise and ordinary multiplication of matrixes and a sign of matrix transposition.

Procedure of the calculation of optimal values of model parameters. According to Bouguer-Lambert- Beer's law, the formula for the calculation of extinction in the matrix form can be written as Eq. (4).

$$
\mathbf{A}_{\text {calc }}=c_{f} \mathbf{E} \text {. }
$$

Here $\mathbf{E}$ is a matrix of molar extinction coefficients at the wavelengths selected for measuring; $\boldsymbol{c}_{f}$ is a matrix of equilibrium concentrations of forms at any ligand concentration. Values of the matrix $\mathbf{E}$ elements were calculated for current values of optimized parameters using the procedure of matrix division [Eq. (5)], which is equivalent to the procedure of multiplying by the pseudo-versed matrix [31].

$$
\mathrm{E}=\left(\mathbf{A}_{\exp } / c_{f}\right)>0 .
$$

Here $\mathbf{A}_{\exp }$ is a matrix of experimental extinction values at selected wavelengths for UV absorption spectra and absolute ellipticity values for circular dichroism (CD) spectra. In the matrix calculated by Eq. (5) negative values were equated to zero, after which it was substituted in Eq. (4). Then the sum of squared deviations (6) of experimental values of absorption $\mathbf{A}_{\text {exp }}$ $\left(\mathbf{F}_{\text {exp }}\right)$ from the corresponding values calculated by the minimization of $\mathbf{A}_{\text {calc }}\left(\mathbf{F}_{\text {calc }}\right)$ was calculated.

$$
S=\Sigma\left(\mathbf{F}_{\text {exp }}-\mathbf{F}_{\text {calc }}\right)^{2} \text {. }
$$

Formula (6) was used also for the determination of matrix model parameters on the basis of diagrams obtained by the ALS-MCR method. In this case the sum of squared deviations of all elements of the concentration matrix calculated by means of ALS-MCR $\boldsymbol{c}_{f}{ }^{\exp }\left(\mathbf{F}_{\exp }\right)$ from the corresponding values calculated in the next iterative step $\mathbf{F}_{\text {calc }}$ by Eq. (3) was minimized. In the present work the standard Levenberg- Marquardt's algorithm of the nonlinear least squares method $[32,33]$ was used to determine the minimum of $S$ by variation of independent variables $\left(K^{-}, \omega_{c}\right.$, and $\left.\omega_{a}\right)$. The optimization was stopped when changes of the model parameters ceased to 
cause appreciable decrease in $S$, which corresponds to the minimum of the calculation error. The error was calculated in the matrix form by Eq. (7).

$$
=\sqrt{\frac{\operatorname{Prace}\left[\left(\mathbf{F}_{\exp }-\mathbf{F}_{\text {calc }}\right) \cdot\left(\mathbf{F}_{\text {exp }}-\mathbf{F}_{\text {calc }}\right)^{T}\right]}{\operatorname{Trace}\left(\mathbf{F}_{\exp } \mathbf{F}_{\text {exp }}^{T}\right)}} .
$$

The hypothesis about the model adequacy was checked by the analysis of homogeneity of the experimental and calculated absorption variance [34]. Tabulated values of Fisher's criterion $F_{\text {tabl }}(\alpha 0.05)$ were used in the check. According to the principle of maximal likelihood, under the condition of homogeneity of variance values, the parameters corresponding to the minimum of the $P E$ function correspond to the most probable model of a system. For the profiles found by means of ALS-MCR, the experimental variance is unknown. Therefore the check of the hypothesis about coincidence of experimental and calculated profiles by the statistical criterion was not carried out in this case.

We have measured values of absorption in the UV spectral range and of signals in the CD spectrum at 101 wavelengths for $33 \mathrm{pH}$ values of a ckit4 aqueous solution. For each $\mathrm{pH}$ value UV and $\mathrm{CD}$ spectra were measured in parallel. Such experimental expedient allows us to construct a stitched matrix of multiinstrumental experimental data of the 33:202 dimension. This matrix was constructed from scaled measured values of the $C D$ spectral signals and from measured absorption $\mathbf{A}_{\exp }=\left[\left(Y_{-} c d\right) / 20, Y \_u v\right]$ values. The graphical representation of the transposed $\mathbf{A}_{\text {Exp }}^{T}$ matrix is the set of measured UV and CD spectra shown in Fig. 1.

Singular value decomposition (SVD) of the $\mathbf{A}_{\text {exp }}$ matrix shows that the data variance can be described within the limits of ordinary experimental accuracy, using three principal components. It is necessary to note that the use of four components does not give rise to the rank deficit problem. On this basis we have analyzed the experimental data matrix in the next step by means of the ALS-MCR procedure of soft simulation in view of 3 and 4 components. The results of the ALS-MCR application are given in Table 1. The distribution diagram calculated for three components is shown in Fig. 2.

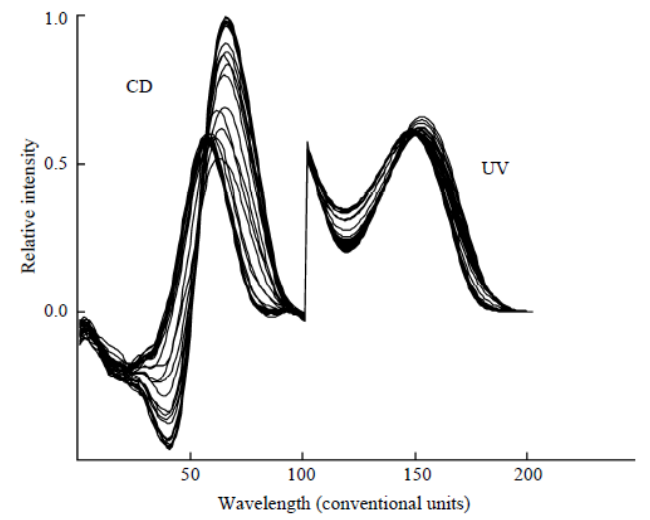

Fig. 1. Experimental UV-VIS absorption spectra $(220-320 \mathrm{~nm}$, at intervals of 1 $\mathrm{nm}$ ) and circular dichroism spectra (220-320 nm, at intervals of $1 \mathrm{~nm}$ ) of ckit4 
solutions at various $\mathrm{pH}$ at $25^{\circ} \mathrm{C}$. Supporting electrolyte $147 \mathrm{mM} \mathrm{K}^{+}(\mathrm{KCl})$ and $1 \mathrm{mM}$ $\mathrm{Mg}^{2+}\left(\mathrm{MgCl}_{2} \cdot 6 \mathrm{H}_{2} \mathrm{O}\right)$. Concentration of ckit4 is $4.3 \mu \mathrm{M}$.

On the basis of the spectra and known data on the ckit4chemical behavior, spectral forms can be assigned to the non-protonated form of polynucleotide I, passing on $\mathrm{pH}$ decrease in the protonated form III having a characteristic structure. As pH decreases further, ckit4 is transformed to the completely protonated coil-like form IV. However for the formation of a stable i-motif structure ckit4 should add two or three protons. Thus, on the ground of chemical reasons, there should be also the monoprotonated ckit4 form II. The presence of the fourth form is confirmed by the presence of a small neglected dispersion at $\mathrm{pH}$ 6-6.5. The result of the decomposition of the data matrix on the assumption that four forms of complexes are formed is shown in Fig. 2. It is seen from the figure that the spectrum profile and the concentration profile of form II contain a very high noise level. Such sensitivity to experimental errors is caused by a low relative content and hence by a selectivity deficit leading to a systematic error in calculations and to rotational uncertainty.

It was noted in the literature that rotational uncertainty is characteristic of all methods of the decomposition of matrixes of the spectrometric analysis data connecting a total instrumental signal with contributions of individual (pure) components of a system [35]. It seems to be one of main reasons for the simulative uncertainty of the spectrophotometric titration data for systems with an incomplete concentration or spectral selectivity. As a rule, in such systems an apparent decrease in the rank of experimental data matrix is observed. To determine more precisely the shape of the form II spectrum and its concentration profile, we have carried out calculations using a hypothesis about a chemical model of system, i.e. taking into account the material balance equation and the law of mass action.

Using the soft-simulation diagrams, we can make a series of conclusions about the character of chemical transformations occurring in the system. Correlating spectral forms with explicit chemical forms allows us to find the $\mathrm{pH}$ range where the ratio of concentrations obeys the law of mass action. According to this law, when several protons are added simultaneously during the formation of a protonated complex, relation (8) should be obeyed.

$$
f=\log ([i-m o t i f] /[\mathrm{Pn}])=\log \left(K_{\text {assoc }}\right)-n \mathrm{pH} .
$$

It follows from the analysis of the experimental data that $i$-motif is formed in the region of $\mathrm{pH} 6$ by simultaneous addition of three protons. The dependence of $\log \left(K_{\text {assoc }}\right)$ on $\mathrm{pH}$ at $\mathrm{n}=3$ is shown in Fig. 3 . It is seen from it that in the region of $\mathrm{pH} 6$ the value of the function is independent of the argument; therefore, in the specified region the model described by Eq. (8) asserts. Using Eq. (8), we have calculated protonation constants and numbers of the protons participating in the transitions. The result of the analysis of diagrams of distribution over forms calculated by ALS-MCR (for three forms with a limitation on unimodality) is presented below. It is necessary to note that the calculated parameters are valid only in narrow $\mathrm{pH}$ ranges. In the conjugated ranges the protonation process is of the 
cooperative or anticooperative character. To describe the titration in the whole studied $\mathrm{pH}$ range, we applied the matrix method.

$\begin{array}{lcc}\text { Transition } & \begin{array}{c}\text { From neutral form to } \\ \text { i-motif }\end{array} & \begin{array}{c}\text { From i-motif to statistical } \\ \text { coil }\end{array} \\ \mathrm{pH} & 6.45-5.70 & 3.84-3.40 \\ \begin{array}{l}\text { Number } \\ \text { of protons }\end{array} & 2.73 \pm 0.06 & 2.05 \pm 0.14 \\ \log \left(K_{\text {assoc }}\right) & 15.9 \pm 0.4 & 7.2 \pm 0.5 \\ R^{2} & 0.997 & 0.980\end{array}$

Originally we have checked up the hypothesis that the variation of spectra is caused by the intercomversion of three spectral forms. Such allowance is valid if the spectrum of a monoprotonated form is strongly correlated with the spectrum of any other form. In this case the concentration profile is the sum of concentration profiles of the forms with correlated spectra. As it is seen from the above data, the protonation parameters calculated from the ALS-MCR diagram and directly from the experimental data are sufficiently close to each other. The found values of the parameters show that the formation of the i-motif complex is a cooperative process, and its protonation is accompanied by the anticooperative effect. It can be explained by the fact that in the stage of the molecule folding in the i-motif structure additional hydrogen bonds are formed in the structure of the complex, which are broken in the second stage when passing in the state of a statistical coil. The discrepancy between the profiles found by means of ALS-MCR and most close to them profiles calculated on the basis of the matrix method is $7.4 \%$. It is necessary to note that the results obtained by the optimization of the matrix of UV absorption are somewhat different. In this case the cooperativity of the $i$-motif complex formation is exhibited not so noticeably, and the anticooperativity of its destruction is much less. The shapes of the spectra found by the expansion of the matrix by the ALS-MCR procedure and by the application of the matrix model considering three forms practically coincide.

Table 1. Results of the MCR-ALS analysis [12] of the experimental data matrix

\begin{tabular}{c|c|c|c|c}
\hline $\begin{array}{c}\text { Number of } \\
\text { components }\end{array}$ & $\begin{array}{c}\text { Error in the description of } \\
\text { experimental data by the method } \\
\text { of principal components, } \%^{\mathrm{a}}\end{array}$ & $\begin{array}{c}\text { Error in the description of } \\
\text { experimental data by MCR-ALS, } \\
\%\end{array}$ & $\begin{array}{c}\text { Standard } \\
\text { deviation }\end{array}$ & $\begin{array}{c}\text { Variance described by the } \\
\text { sum of principal components, } \\
\%\end{array}$ \\
\hline 2 & 5.33 & 5.41 & 0.56 & 99.71 \\
3 & 1.29 & 1.50 & 0.16 & 99.98 \\
$3 \mathrm{u}^{\mathrm{b}}$ & 1.29 & 1.54 & 0.16 & 99.98 \\
4 & 1.15 & 1.33 & 0.14 & 99.98 \\
$4 \mathrm{u}^{\mathrm{b}}$ & 1.15 & 1.56 & 09.98 \\
\hline a $f(\%)=100 \cdot \sqrt{\sum_{i j}^{2} \sum_{i j}^{2} d_{i j}^{2}}\left(r_{i j}\right.$ are differences between experimental data $d_{i j}$ and values calculated for the matrix A $=c$ E reproduced using a \\
specified number of components. ${ }^{b}$ With the one-modality limitation.
\end{tabular}




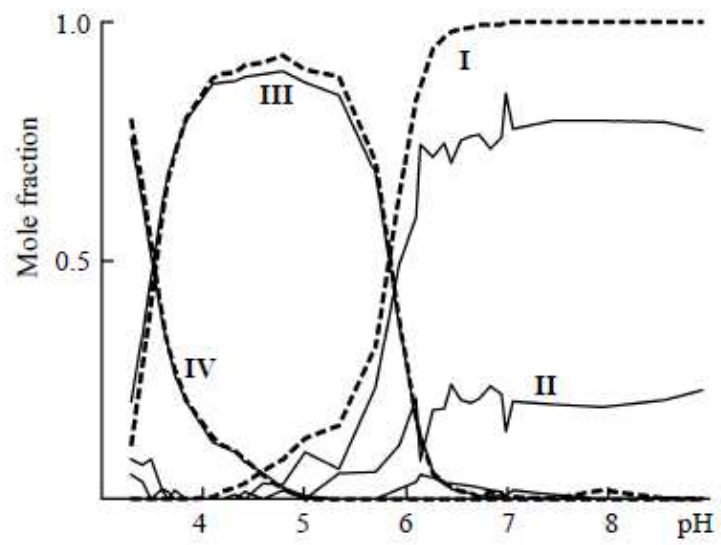

Fig. 2. Relative contents of ckit4 forms as functions of $\mathrm{pH}$ found by ALS-MCR (solid lines) in view of 4 spectral forms and (dashed lines) in view of 3 spectral forms. (I) non-protonated form of the polynucleotide, (II) monoprotonated form, (III) form with $i$-motif structure, (IV) completely protonated coil-like form.

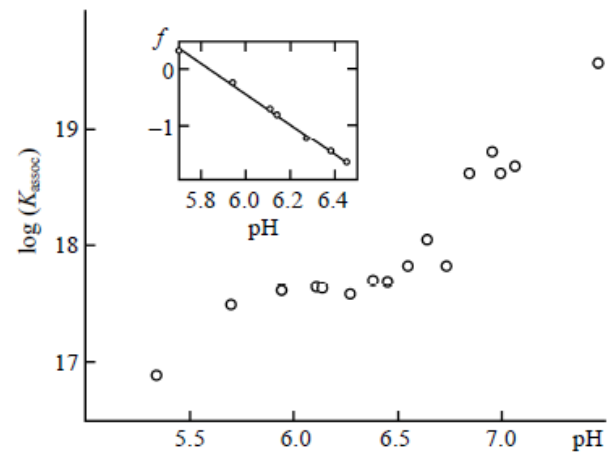

Fig. 3. Dependence of apparent protonation constant of ckit4 on $\mathrm{pH}$ of solution. (Cycles) denote log $\left(K_{\text {assoc }}\right)$ values calculated by equation $(8)(n=3)$ from the results of the decomposition of the experimental data matrix using ALS- MCR. The dependence of $f$ on $\mathrm{pH}$ calculated by the leastsquares method in the $\mathrm{pH}$ range 5.765.45 is shown in the inset.

Table 2. Parameters of ckit4 protonation in aqueous solution at $\mathrm{pH}$ 3.29-8.93.

\begin{tabular}{l|l|c|c|c|c}
\hline \multicolumn{1}{c|}{ Model } & $\begin{array}{c}\text { Target } \\
\text { function }\end{array}$ & $\log K_{\text {in }}$ & $\omega_{c}$ & $\omega_{a}$ & $P E$ \\
\hline ALS-MCR (3s) & $A_{\exp }$ & - & - & - & 1.95 \\
Matrix (3s) & $c_{f(A L S-U)}$ & 4.86 & 1.33 & 0.049 & 7.4 \\
Matrix (3s) & $A_{\operatorname{exp~(UV)~}}$ & 5.07 & 1.15 & 0.075 & 1.13 \\
Matrix (3s) & $A_{\operatorname{exp~(CD)~}}$ & 4.25 & 2.08 & 0.0069 & 4.72 \\
Matrix (3s) & $A_{\exp }$ & 4.30 & 2.01 & 0.0080 & 3.33 \\
ALS-MCR (4s) & $A_{\exp }$ & - & - & - & 1.77 \\
Matrix (4s) & $c_{f(A L S)}$ & 4.87 & 1.34 & 0.046 & 19.8 \\
Matrix (4s) & $A_{\operatorname{exp~(UV)~}}$ & 5.36 & 1.00 & 0.12 & 1.01 \\
Matrix (4s) & $A_{\exp (C D)}$ & 4.48 & 1.75 & 0.015 & 4.96 \\
Matrix (4s) & $\boldsymbol{A}_{\exp }$ & 4.52 & 1.71 & 0.016 & 3.45 \\
\hline
\end{tabular}


It follows from the ALS-MCR data that the addition of the fourth component in the matrix expansion actually leads to the splitting of the form dominating in neutral and weakly acid solutions. Using the matrix model, as it was the case with three forms, we have optimized various target functions. The resulting protonation parameters are presented in Table 2. The use of four forms improves the description of only UV absorption matrix. The error in the description of the CD data matrix slightly increases. Similarly to the use of three forms for the description, a certain difference is observed in the results obtained by the optimization of the UV absorption matrix. In this case cooperatively of the $i$-motif formation is completely not exhibited.

The diagram of ckit4 distribution by forms of complexes is shown in

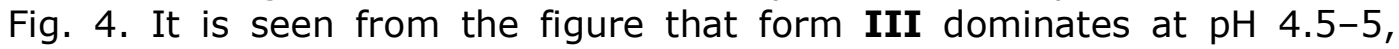
therefore this field can be considered as the concentration-selectivity field. There are no concentration selectivity regions for forms II and IV. The absorption spectra of individual forms of complexes calculated using ALSMCR and the matrix model are shown in Fig. 5a. The spectra calculated by the both by methods have an equal shape in spite of the absence of concentration selectivity. A hypochromic shift is observed on passing from form IV to form III, as well as on the (II $\rightarrow \mathbf{I}$ ) passing. As known, this effect is observed on passing from a statistical coil state to an ordered structure if it is results in a parallel orientation of the moments of the $\Pi-\Pi^{*}$ quantum transitions located in the plane of nucleic bases [36]. We assume on this basis that, as a result of the first proton addition, the oligomer molecule loses its original structure and probably takes a bend, which makes easier the formation of the i-motif structure upon the further protonation. The CD spectra found for separate forms are shown in Fig. 5. In this case owing to the absence of concentration selectivity the spectrum of forms II found by ALS-MCR has an irregular shape. In the matrix model this spectrum has a smoothed appearance that has a clearer physical sense. It is seen from the figure that the spectra of forms I, II, IV are similar to each other. The spectrum of form III differs from them by much higher values of molar ellipticity both in the region of a minimum and in the region of a maximum, i.e. the absorption intensities for counterclockwise-polarized and clockwise- polarized light strongly differ from each other, which is a characteristic indication of the formation of the special $i$-motif structure.

In conclusion we can note that scheme developed in the present work for the mathematical analysis of changes in the absorption and circular dichroism spectra occurring upon $\mathrm{pH}$ variation in the aqueous solution of a DNA oligonucleotide has allowed us to understand chemical stages of the protonation process more deeply. The combination of soft and rigid simulation has made it possible to determine not only spectral forms and abstract diagrams of their concentration distribution, but also their chemical nature, and to calculate the spectra of particular chemical components of the system under study. A good coincidence of experimental and calculated data suggests that the offered scheme of the analysis can be further effectively used for the description of similar systems. 


\section{EXPERIMENTAL}

The nucleotide sequence 5'-CCTTTCCTTTTCCTTTCC-3' was synthesized with the aid of an automatic DNA synthesizer (Applied Biosystems Mod. 392), using standard 2-cyanoethyl phosphoramidites. The oligonucleotide was purified with a cartridge according to the producer instruction. Desalting of the reaction product was carried out using a Sephadex G-25 column. The reagents $\mathrm{KCl}, \mathrm{MgCl}_{2} \cdot 6 \mathrm{H}_{2} \mathrm{O}, \mathrm{HCl}$, and $\mathrm{NaOH}$ were purchased from Panreac (Spain). To prepare solutions, MilliQ ${ }^{\circledR}$ water was used.

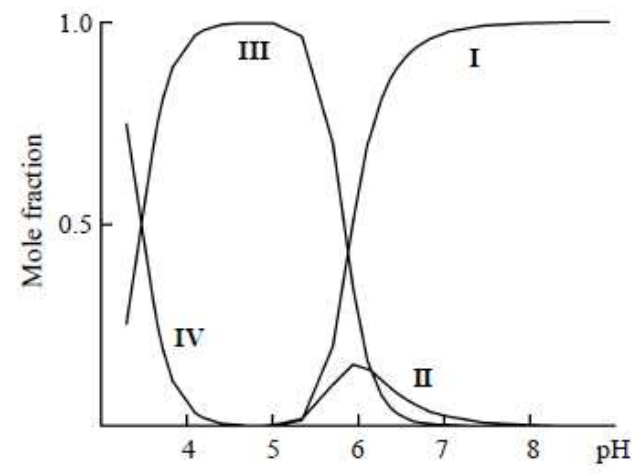

Fig. 4. Relative contents of ckit4 forms at various $\mathrm{pH}$ found by the matrix method in view of 4 spectral forms. (I) non-protonated form of the polynucleotide, (II) monoprotonated form, (III) form with i-motif structure, and (IV) completely protonated coil-like form.

(a)

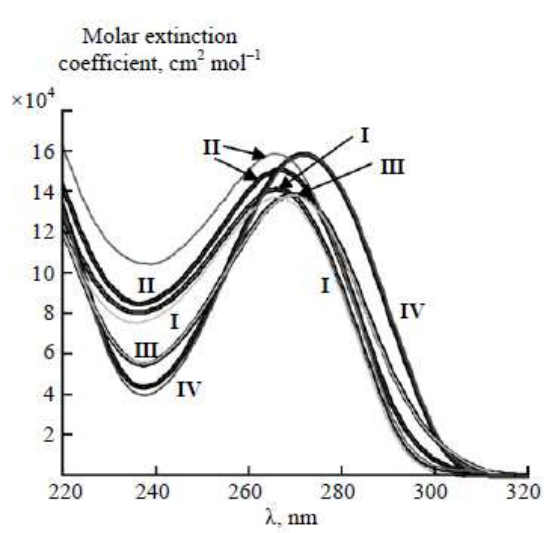

(b)

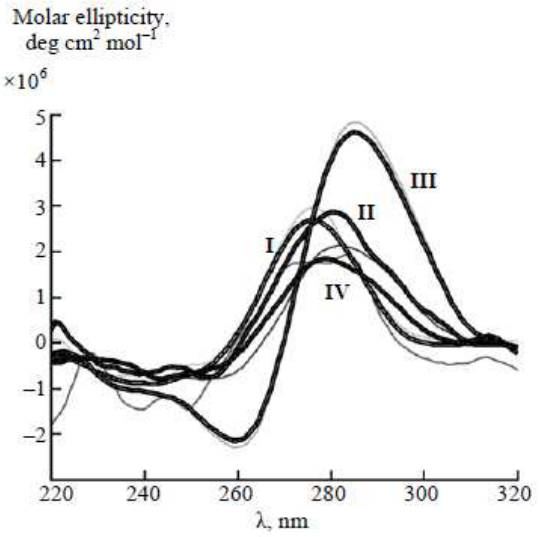

Fig. 5. (a) UV and (b) CD spectra of individual spectral forms calculated by ALSMCR are denoted by thin lines. Spectra calculated by the matrix model are denoted by solid heavy lines. (I) non-protonated form of the polynucleotide, (II) monoprotonated form, (III) form with i-motif structure, and (IV) completely protonated coil-like form.

Electronic absorption spectra were measured on an HP8453 UV spectrophotometer. While measuring spectra temperature was maintained by means of a device based on Peltier effect. Circular dichroism spectra 
were measured on a Jasco J-810 spectro-polarimeter equipped with a Julabo F-25/HD device for maintaining a specified temperature. Quartz cells with the optical path length of $1 \mathrm{~cm}$ and volume of $3000 \mu \mathrm{l}$ or $1500 \mu \mathrm{l}$ (Hellma) were used. Measurements of $\mathrm{pH}$ were carried out with an Orion SA $720 \mathrm{pH} / \mathrm{ISE} \mathrm{pH}$-meter and a combined microelectrode (Thermo) at $25^{\circ} \mathrm{C}$ and the solution ionic strength of $150 \mathrm{mM}\left(147 \mathrm{mM} \mathrm{K}^{+}\right.$in the form of $\mathrm{KCl}$ and $1 \mathrm{mM} \mathrm{Mg}{ }^{2+}$ in the form of $\mathrm{MgCl}_{2} \cdot 6 \mathrm{H}_{2} \mathrm{O}$ ). The titration was carried out by adding microamounts of a concentrated $\mathrm{NaOH}$ solution to an oligonucleotide solution acidified by $\mathrm{HCl}$. The concentration of DNA was measured by the absorption at $260 \mathrm{~nm}$ using the technique described in [36]. Solutions with the oligonucleotide concentration of $4.3 \mu \mathrm{M}$ were prepared for the titration.

\section{REFERENCES}

1. http: en.wikipedia.org/wiki/Cooperativity.

2. McGhee, J.D. and von Hippel, P.H., J. Mol. Biol., 1974, vol. 86, no. 2, p. 469.

3. Scatchard, G., Ann. New York. Acad. Sci., 1949, vol. 51, p. 660.

4. Crothers, D.M., Biopolymers, 1968, vol. 6, no. 4, p. 575.

5. Zasedatelev, A.S., Gurskii, G.V., and Vol'kenshtein, M.V., Molek. Biol., 1971, vol. 5, no. 2, p. 245.

6. Hill, T.L., Cooperativity Theory in Biochemistry. Steady State and Equilibrium Systems, New York: Springer Verlag, 1985.

7. Kudrev, A.G., Vysokomol. Soed., Series A, 2000, vol. 42, no. 5, p. 803.

8. Jaumot, J., Vives, M., and Gargallo, R., Anal. Biochem., 2004, vol. 327, p. 1.

9. Jaumot, J., Eritja, R., Tauler R., and Gargallo R., Nucleic Acids Res., 2006, vol. 34, p. 206.

10. Vives, M., Tauler, R., Eritja, R., and Gargallo, R., Anal. Bioanal. Chem., 2007, vol. 387, p. 311.

11. Khan, N., Aviñó, A., Tauler, R., González, C., Eritja, R., and Gargallo, R., Biochimie, 2007, vol. 89, p. 1562.

12. http:www.ub.edu/mcr/ntheory.htm.

13. Gehring, K., Leroy, J.L., and Gueron, M., Nature, 1993, vol. 363, p. 561.

14. Ahmed, S., Kintanar, A., and Henderson, E., Nat. Struct. Biol., 1994, vol. 1, p. 83.

15. Leroy, J.L., Gehring, K., Mergny, J.L., and Hélène, C., Nucleic Acids Res., 1994, vol. 22, p. 1600.

16. Gueron, M. and Leroy, J.L., Current Opinion in Struct. Biol., 2000, vol. 10, p. 326.

17. Mergny, J-L., Lacroix, L., Han, X., Leroy, J-L., and Helene, C., J. Am. Chem. Soc., 1995, vol. 117, no. 35, p. 8887.

18. Cancer Chemotherapeutic Agents, William, O.F., Ed., Washington D.C.: ACS Prof. Ref. Book, 1995.

19. Seela, F. and Budow, S., Helv. Chim. Acta, 2006, vol. 89, p. 1978.

20. Sharma, J., Chlabra, R., Yan, H., and Liu, Y., Chem. Comm., 2007, vol. 5, p. 477.

21. Li, X., Peng, Y., Ren, J., and Qu, X., Proc. Natl. Acad. Sci. USA, 2006, vol. 103, p. 19658. 
22. Hurley, L., Bialis, T., Dexheimer, T.S., Sun, D., and Gleason-Guzman, M., Abstracts of Papers, 19th Rocky Mountain Regional Meeting of ACS, 2006, RM-251.

23. Xu, Y. and Sugiyama, H., Nucleic Acids Res., 2006, vol. 34, p. 949.

24. Gampp, H., Maeder, M., Meyer, C.J., and Zuberbuhler, A.D., Talanta, 1985, vol. 32, no. 12, p. 1133.

25. Gampp, H., Maeder, M., Meyer, C.J., and Zuberbuhler, A.D., Talanta, 1986, vol. 33, no. 12, p. 943.

26. Gampp, H., Maeder, M., Meyer, Ch., and Zuberbuhler, D., Anal. Chim. Acta., 1987, vol. 193, no. 1, p. 287.

27. Kudrev, A. and Tauler, R., Abstracts of Papers, Euroanalysis IX: European Conference on Analytical Chemistry, Bolonga (Italy), 1996, p. 160.

28. Kudrev, A., Tauler, R., and Izquierdo-Ridorsa, A., Abstracts of Papers, $V$ Scandinavian Symposium on Chemometrics, Lahti, 1997, p. 65.

29. Kudrev, A.G., Zh. Obshch. Khim., 2003, vol. 73, no. 1, p. 23.

30. Golub, G.H. and Van Loan, C.F., Matrix Computations, London: Johns Hopkins Univ. Press, 1989.

31. Marquardt, D.W., J. Soc. Ind. Appl. Math., 1963, vol. 2, no. 2, p. 431.

32. Maeder, M. and Zuberbuhler, A.D., Analyt. Chem., 1990, vol. 62, p. 2220.

33. Massart, D.L., Vandeginste, B.G.M., Deming, S.N., Michotte, Y., and Kaufman, L., Chemometrics: A Textbook, Amsterdam: Elsevier Publ., 1988.

34. Tauler, R. and de Juan, A., in Practical Guide to Chemometrics, ch. 11, 2nd ed., Taylor and Francis Group, LLC, 2006.

35. Seitai Kobunshi (Biopolymers), Imanishi, Y., Ed., vol. 14, Tokyo: Kyoritsu Shuppan, 1985.

36. Allawi, H.T. and SantaLucia, J., Biochemistry, 1997, vol. 36, p. 10581. 